\title{
Interhospital Transfers for Quality Assessment of Healthcare Systems
}

\author{
Scott A Lorch, MD, MSCE ${ }^{1,2 *}$
}

'Department of Pediatrics, The Children's Hospital of Philadelphia, Perelman School of Medicine, University of Pennsylvania, Philadelphia, Pennsylvania; ${ }^{2}$ Senior Scholar, Leonard Davis Institute, University of Pennsylvania, Philadelphia, Pennsylvania.

W ith the increasing percentage of our gross national product being allotted to healthcare and concerns about the care received by patients, the number of measures to assess the quality and efficiency of care delivered by healthcare professionals has increased. The paper by Mueller et al. ${ }^{1}$ adds to our understanding of an important yet relatively understudied group of patients: those that require transfer from one inpatient facility to another. In general, these patients are sicker and exhibit poor outcomes, especially with time-sensitive management conditions, such as cerebrovascular accidents, or conditions where the transfer itself may cause harm to the patient, such as the case of an infant born prematurely. However, transferring patients with less time-dependent conditions may not be associated with such negative results. ${ }^{1}$ The uniqueness of interhospital transfers is attributed to their ability to provide insights into the care practices of other actors within the healthcare system, namely, the transferring hospital and the larger healthcare system, and to describe how the care quality may change in hospitals during periods of stress, such as during overcrowding or high patient acuity.

As described by Mueller et al. the care and outcomes of patients transferred to a hospital may provide information regarding the key aspects of care at the receiving hospital; these aspects include the capability for triage of potentially high-acuity patients and the management of such patients during periods of crowding and organizational stress. These measures of efficiency have rarely been studied in relation to the care provided to patients and their ultimate outcomes. The most studied efficiency measure is hospital crowding, which has been shown in numerous studies to be associated with lower efficiency as measured by the length of stay, lower quality of care, and higher mortality. ${ }^{2-3}$ This report by Mueller et al. is one of the first papers to highlight how other aspects of the care delivery system, including the triage practices and the response of a hospital system to stress, may influence care outcomes. The limitation of other studies in exploring the relationship between the measures of efficiency and quality of care, as noted by a systematic review of healthcare efficiency measures by Hussey et al. ${ }^{4} \mathrm{em}-$ phasizes the need to understand the drivers of low quality of care and to determine the specific times at which such care may be compromised by other factors, such as patient volumes.

*Corresponding Author: Scott A. Lorch, MD, MSCE; E-mail: lorch@email.chop. edu; Telephone: 267-259-5534

Received: May 7, 2019; Accepted: May 8, 2019

() 2019 Society of Hospital Medicine DOI 10.12788/jhm.3243
Although interhospital transfers may offer certain insights into the efficiency of care delivered at the hospitals receiving these patients, they are generally rare and centered on a few quaternary hospitals within a region. ${ }^{3}$ In addition, the Mueller paper reveals that not all these transfers have high disease acuity, particularly for cardiac patients. Whether claims-based approaches to risk adjustment would sufficiently differentiate the reasons for the transfer/failure to transfer of patients is unclear and thus may be affected by the selection bias. With these issues, the outcome of transferred patients may be only of limited value when assessing the care quality of hospitals that generally receive transferred patients from other medical institutions within a given geographic area. ${ }^{5}$

Interhospital transfers may provide insights into the care of patients at the hospitals which transfer out such patients, focusing on the appropriateness of transfers, how these hospitals operate when such a sick patient arrives at that hospital, and the outcomes of patients with conditions that may require transfer. A few studies have explored the preventable transfer, particularly for trauma patients, where a preventable transfer was defined as a transfer that was was not admitted to the receiving hospital and did not receive any procedures or testing. Although not readily defined for numerous conditions, such a measure would provide insights into how hospitals decide whether a patient requires care at a higher-level hospital and assessing the processes needed to optimize this decision-making process, including where the patient ultimately is transferred. In a study of patients with acute myocardial infarction, $36.8 \%$ of cases that required transfer were not directed to hospitals with the best outcomes as measured by 30-day risk-adjusted mortality rates within a given geographic region. ${ }^{6}$ Such decisions would contribute to the potential worse outcomes observed in patients requiring interhospital transfer.

Finally, transfers provide insights into the functioning of the larger healthcare system. The measures assessing the functioning of the healthcare system are rare. In theory, interhospital transfers meet the goals of a functioning regional healthcare system by matching the patients to facilities with the suitable capabilities to manage the patient's given type of illness or injury. Such a system, however, requires collaboration between hospitals who otherwise compete for patients. The literature suggests that such collaboration is widely variable and dependent on patient factors, such as the types of conditions and their insurance status, ${ }^{7}$ and the costs required by hospitals to add the services needed to care for increasingly ill patients. In addition, the growth of so-called narrow insurance networks, which limit the number of hospitals an insurance company will 
include on their preferred network, may place barriers on the appropriate location of such transfers based on the quality of the receiving hospital. ${ }^{8}$

The paper by Mueller et al. adds to the literature the unique aspects of the care needed by the patients requiring interhospital transfer. Unlike most other measures of care quality and efficiency, interhospital transfers potentially offer knowledge about the quality of the larger healthcare system, assessing the appropriateness and ultimate outcomes not only of patients who are transferred but similarly sick patients who could have potentially benefited from a transfer and how the actors within the system may respond to periods of high patient load and stress. By understanding the drivers of the appropriateness of where patients receive care, we can gain insights into the mechanisms needed to fulfill the goals of a functional regionalized healthcare system.

Disclosures: The author has no financial or other relevant conflicts of interest to disclose.

\section{References}

1. Mueller SK, Fiskio J, Schnipper J. Interhospital transfer: transfer processes and patient outcomes. J Hosp Med. 2019;(8):486-491.

2. Lorch SA, Millman AM, Zhang X, Even-Shoshan O, Silber JH. Impact of admission-day crowding on the length of stay of pediatric hospitalizations. Pediatrics. 2008;121(4):e718-e730. https://doi.org/10.1542/peds.2007-1280.

3. Sun BC, Hsia RY, Weiss RE, et al. Effect of emergency department crowding on outcomes of admitted patients. Ann Emerg Med. 2013;61(6):605-611.e6. https://doi.org/10.1016/j.annemergmed.2012.10.026

4. Hussey PS, de Vries H, Romley J, et al. A systematic review of health care efficiency measures. Health Serv Res. 2009;44(3):784-805. https://doi. org/10.1111/j.1475-6773.2008.00942.x.

5. Lorch SA. National quality measures in perinatal medicine. Clin Perinatol. 2017;44(3):485-509. https://doi.org/10.1016/j.clp.2017.05.001

6. Iwashyna TJ, Kahn JM, Hayward RA, Nallamothu BK. Interhospital transfers among Medicare beneficiaries admitted for acute myocardial infarction at nonrevascularization hospitals. Circ Cardiovasc Qual Outcomes. 2010;3(5):468-475. https://doi.org/10.1161/CIRCOUTCOMES.110.957993.

7. Green A, Showstack J, Rennie D, Goldman L. The relationship of insurance status, hospital ownership, and teaching status with interhospital transfers in California in 2000. Acad Med. 2005;80(8):774-779. https://doi. org/10.1097/00001888-200508000-00015

8. Colvin JD, Hall M, Thurm C, et al. Hypothetical network adequacy schemes for children fail to ensure patients' access to in-network children's hospital. Health Aff (Millwood). 2018;37(6):873-880. https://doi.org/10.1377/ hlthaff.2017.1339. 